\title{
Application Research of Intelligent Technology in Electrical Engineering Automation Control
}

\author{
Jingxin Wang \\ North China Electric Power University, Baoding, 071000, China
}

Keywords: Intelligent technology, Electrical engineering automation, Application research

\begin{abstract}
Science and technology are the technical conditions for the development of intelligent technology, artificial intelligence theory is its theoretical basis, and the need to save labor costs and social costs is a social factor. Under the influence of these comprehensive factors, intelligent technology has increasingly penetrated into all aspects of society, affecting people's lifestyles. Based on this background, this paper takes the application of intelligent technology in electrical engineering automation control as the research topic, cuts in from the development background and theoretical basis of intelligent technology, and conducts four levels of application analysis on the basis of value analysis.
\end{abstract}

\section{Introduction}

The continuous progress of the society and the continuous development of the economy have made the labor force tense and doubled in value, and the cost of various products has risen steadily. This brings new challenges to the production and development of enterprises, and also provides us with excellent opportunities to achieve the reduction of social costs with the help of science and technology. Through the unremitting efforts and research innovation of scientists, the current intelligent technology is more practical and comprehensive, the model construction is simpler and more convenient, and the system is more intelligent. The application of intelligence in various fields of society and various industries is becoming more and more extensive, and its influence is getting deeper and deeper, especially in the automation control of electrical engineering, which greatly reduces labor expenditure and production costs. This article mainly includes three main parts. The first part introduces the concept and theoretical basis of intelligent technology; the second part makes a detailed analysis of the application value and significance of intelligent in electrical engineering; on this basis, combined the case analysis and interpretation of the smart grid.

\section{Theoretical basis of applying intelligent technology to electrical engineering automation control}

\subsection{Overview of intelligent technology}

Intelligentization is an inevitable trend of social development and an inevitable requirement for the realization of quality and efficient development of the whole society. With the continuous improvement of people's living standards and living conditions, their demand for intelligence is becoming stronger and stronger. At present, modern communication technology and computer network technology are developing rapidly, and intelligence has been deeply integrated in various fields of society. The combination of intelligent technology and related fields has greatly saved social costs. Intelligent technology refers to the centralized application of modern communication and information technology, computer network technology, industrial technology, and intelligent control technology. Specifically, intelligent technology is a technology, method and system that uses computer application systems to simulate the principles of human intelligence to perform voice recognition, image recognition, expert command and other operations on industrial production. It has been widely used in industrial production. 


\subsection{Theoretical basis}

Intelligent technology encompasses a wide range of subjects, including informatics, linguistics, and control science. The combination of these theoretical knowledges constitutes the basic theory of intelligence. Moreover, in the course of its operation, the theory of artificial intelligence is further integrated into computer technology, becoming the most advanced scientific technology. After many studies and experiments, researchers have given intelligent technology robots with artificial intelligence, which is usually used in many high-risk jobs. The application of artificial intelligence in electronic information technology has a broad space. Intelligent technology includes electrical and electronic technology, while also collecting information and processing information. As an important part of industrial production, electrical engineering automation control needs to continuously improve work efficiency and value content in order to effectively improve the economic benefits of industrial production. In the automation control of electrical engineering, strengthening the application of intelligent technology helps to improve the automation control capability and control level of the industrialized production process, and improve the efficiency and quality of electrical engineering implementation.

\section{The application value and advantages of intelligent technology in electrical engineering automation control}

The application value of intelligent technology in electrical engineering automation control is mainly reflected in the following aspects.

\subsection{One is to promote the unification of electrical engineering automation control}

The traditional electrical engineering automation control equipment has few control surfaces and limited functions, and can only be controlled specifically for a part. There is no matching facility to effectively control the entire electrical project, so there are several links that require several special controls model. In this regard, intelligent technology has conducted innovative research to reduce the number of control models, and the reduction in number has improved the ability to control electrical engineering automation control systems, and has achieved unified and effective management of electrical engineering automation. Intelligent technology can well manage the designated control objects and non-designated objects, ensuring a comprehensive and unified management mode for electrical engineering automation control.

\subsection{The second is to improve the level of electrical engineering automation control}

With the help of intelligent technology, it is possible to achieve continuous self-improvement and continuous optimization and improvement of electrical engineering automation control technology, thereby achieving value growth. Intelligent technology can improve the level of electrical engineering automation control. Specifically, the operation process of intelligent technology can make the work data in electrical engineering-related equipment detailed and achieve quick feedback to the center. Calculate the parameters and time changes consistent with the equipment, and respond in a timely manner according to the calculation results, issue control commands, and adjust the control strength of electrical engineering automation equipment accordingly. Intelligent technology greatly reduces the time for building a control model, and quickly grasps control data and efficiency. Once problems are found, they are quickly attacked and quickly resolved to ensure the normal operation of equipment and improve the electrical engineering automation control capabilities.

\subsection{The third is to promote a simpler electrical engineering automation model}

Using traditional technology to control electrical engineering, the pre-engineering is very tedious but also very important. First, the parameters in the equipment are calculated and integrated to provide corresponding data for the construction model. Only after the data is obtained can the design of the model be started. The design template also needs to set up corresponding dynamic equations or functions. Only in this way can data control work be carried out, and it can react in case of abnormal conditions. The control model of the traditional control technology appears to be 
particularly rigorous on the surface, but it is not true. Errors and unexpected events occur from time to time, resulting in low reliability of data transmission, and the risk is thus formed. For the above problems, intelligent technology can successfully prevent and avoid. Because there are few control models built by intelligent technology, the interference and influence of these factors on the control process are greatly reduced, and problems are discovered in time and dealt with quickly.

\section{Specific application practice of intelligent technology in electrical engineering automation control}

As the research of intelligent technology becomes more and more mature, it has the basic conditions for application in a variety of complex fields. The application scope of intelligent technology in electrical engineering automation control is more and more extensive, and its application effect and value are more and more prominent.

\subsection{Operational application}

The key to improving the level of electrical engineering automation control lies in the deep intervention of intelligent technology, which is also the inevitable development trend of electrical engineering automation control in the future. The application of intelligent technology in electrical engineering automation and control saves manpower and contributes to efficient work. These advantages and functions make the road of intelligent technology wider and wider, and play a good role in electrical engineering automation and control. The application of intelligent technology in electrical engineering automation control can realize the remote, unmanned, autonomous and efficient operation of the control system. With the help of intelligent technology, the unmanned operation simplifies the control steps and improves the accuracy of work. With the further improvement of the level of intelligent technology, it can also better improve the automation control effect and efficiency of electrical engineering in more fields, which has never brought the transformation of economic benefits of industrial production and the rapid improvement of the core competitiveness of enterprises.

\subsection{Self-diagnosis application}

During the operation of the electrical engineering system, due to the interference of the external environment, the equipment itself, and improper operation and other factors, equipment operation failure problems may occur in some cases. The role of transformers in electrical equipment is very important. During the operation, attention should be paid to the daily maintenance and inspection of the transformers, and they must be inspected regularly and regularly to prevent transformer failures. How to effectively monitor these phenomena requires the introduction of intelligent technology. On the one hand, it can track and record from time to time, on the other hand, it can make a comprehensive judgment on the status quo before the failure. Using intelligent technology, a comprehensive and systematic survey and inspection of many electrical equipment can be carried out and processed in a timely manner. Using intelligent technology to carry out precise inspection of intelligent technology, you can find the problems in the electrical engineering system at the first time and alarm in time, develop the location of the problem in time, gradually narrow down the fault scope, and automatically repair and maintain, reducing electrical equipment The frequency of failure.

\subsection{Design optimization application}

The design scheme of electrical equipment has a great influence on the operation process of automatic control. Using traditional methods to design the automatic control of electrical equipment is a fairly complex process, and the requirements for technicians are also quite high. Only by proficiently grasping the rich and comprehensive subject knowledge and comprehensive application ability of the subject, combined with rich practical experience, can we master and apply intelligent technology to optimize the electrical equipment effectively. With its own capabilities in intelligent perception, agile thinking, and quick behavior, intelligent technology can give full play to its advantages in the application of electrical engineering automation control, effectively strengthen the 
level of automation control in electrical engineering, and realize the safety of electrical engineering. Using intelligent technology and computer system software, you can easily achieve the optimal design of electrical engineering automation control schemes, without wasting too much manpower and time on some details, and it has strong practicality and efficiency.

\subsection{Specific practical applications in the power grid}

With the help of modern automatic control theory and management methods, smart grid has relatively objective development in power grid fault handling, equipment service life extension, and power grid construction cost reduction. Automatic control technology plays an extremely important role in the transmission of smart grid.

One is to close the transmission line fault treatment. The smart grid can centrally debug faulty lines, and relays collectively protect a large number of grid nodes, so its fault tolerance should be strengthened. The application of intelligent technology in it has important significance for the switch control of the trunk line and the use of the line itself. Intelligent technology can realize the division of the main line and the branch line within a short period of time, which is convenient for removing the fault and ensuring the normal operation of the entire power grid.

The second is about intelligent protection of transformers. The transformer controls the efficient operation of the line voltage, combines the transient steady-state operation of the line voltage with the protection of the transformer, and runs through the maintenance process of the smart grid. Applying intelligent technology to the protection of power grid transformers, the voltage can be adjusted within a relatively short period of time in the event of a sudden failure of the voltage limit and specific lines, and can be adjusted by an automatic control system in an emergency to ensure its stable operation.

The third is to improve data processing capabilities. Through the optical cable, the smart grid automation system controls the power grid. In the complete operation process, data generation and synchronization play an extremely important role. It is extremely important to strengthen the data analysis and preprocessing of power grid protection equipment. The application of smart new technologies in information has greatly changed the method of signal generation and transmission. Through network sharing, the construction of internal systems of power stations is becoming more and more suitable for smart grids, which greatly improves on the basis of promoting smart grids to achieve benign meta-transfer Improvement of power grid information and data.

\section{Conclusion}

With the continuous development of Internet technology, the development of globalization is getting deeper and deeper, and the development of science and technology plays a decisive role. Intelligence is an important achievement in the development of science and technology, and its theoretical basis is artificial intelligence. In the context of the rapid development of artificial intelligence, intelligence must play an important role in promoting social development, and the scope of application of intelligent technology continues to expand. In industrial design, electrical engineering automation control realizes unified deployment and management, which greatly reduces a lot of unnecessary costs. At this stage, China's intelligent technology is still in a weak position, or in the development stage. Therefore, only through continuous efforts to learn, learn from the experience of some advanced technologies, enrich knowledge, and establish a good foundation, can China's intelligent technology leapfrog development, and then make China a high-tech technological power.

\section{References}

[1] Linlin Li, Mohammed Chadli, Steven X. Ding,. Diagnostic Observer Design for T-S Fuzzy Systems: Application to Real-Time-Weighted Fault-Detection Approach[J]. IEEE Transactions on Fuzzy Systems, 2018, 26(2):805-816.

[2] Endryansyah, Rusimamto P W, Ridianto A, et al. Development of Servo Motor Trainer for Basic 
Control System in Laboratory of Electrical Engineering Control System Faculty of Engineering Universitas Negeri Surabaya[J]. 2018.

[3] Jin Wang, Hua Shao. Application of Wireless Sensor Network Technology in Security Control of Intelligent Buildings[J]. International Journal of Online Engineering, 2018, 14(5):93.

[4] Li Bin, Long Yuan, Liu Huan. Research progress on Terahertz technology and its application in agriculture[J]. Transactions of the Chinese Society of Agricultural Engineering, 2018.

[5] Na Wang, Jinguo Wang, Yuanyuan Wang. The Application of Intelligent Control in Clinical Medicine[J]. MATEC Web of Conferences, 2019, 267(1):02006.

[6] Fei-Yue Wang, Dimitar P. Filev, Witold Pedrycz,Guest Editorial From Intelligent Control to Smart Management of Cyber-Physical-Social Systems: A Celebration of 70th Anniversary of Cybernetics by Norbert Wiener[J]. IEEE TRANSACTIONS ON CYBERNETICS, 2018. 